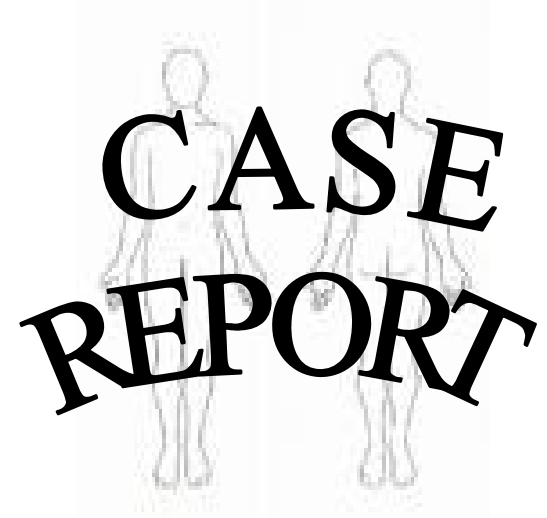

\section{Conservative Management of Spontaneous Bladder Rupture}

Wissam Saliba, M.D. ${ }^{1}$,

Michael E. Grant, M.D. ${ }^{2,3}$

${ }^{1}$ University of Wisconsin

School of Medicine and Public Health,

Department of Medicine, Madison, WI

${ }^{2}$ University of Kansas

School of Medicine-Wichita, Department of Internal Medicine

${ }^{3}$ Wichita Nephrology Group, PA, Wichita, KS

\section{Introduction}

Spontaneous rupture of the bladder (SRUB) is an uncommon disease. The cornerstone of management is a high index of suspicion. In an era where alcohol intoxication is common, SRUB should always be in the differential diagnoses of acute abdominal pain, inability to void, and ascites. We report a case of SRUB secondary to alcohol intoxication presenting with elevated serum creatinine and abdominal ascites. Although reparative surgery was performed in most reported cases, conservative management was successful in this case.

\section{Case Report}

A 29-year-old, previously healthy man was admitted to the hospital for oliguric acute kidney injury. He had been intoxicated with alcohol the day prior to presentation. He reported having lost consciousness and passing out on the kitchen floor for more than seven hours. Upon awakening, he had abdominal pain and low urine output over the next 24 hours.

He presented to the emergency department where he received four liters of normal saline and subsequently was admitted to the hospital with an elevated creatinine of $4.86 \mathrm{mg} / \mathrm{dL}$. He complained of mild diffuse abdominal pain, but denied hematuria or dysuria. He denied any recent nonsteroidal anti-inflammatory drug use.

His past medical history was negative. He smoked cigarettes and did not report any illicit drug use. He drank alcohol inconsistently, but had a tendency to binge drink every 2-3 weeks.

On physical examination, his vital signs were normal. His abdomen was tender, but soft to palpation. He had a positive wave sign, indicating the presence of ascites.

His white blood cell count was 13,700 $\mathrm{ml}$ with $80 \%$ neutrophils. His complete metabolic profile revealed a serum sodium level of $130 \mathrm{mEq} / \mathrm{L}$, creatinine of 4.86 $\mathrm{mg} / \mathrm{dL}$, and BUN of $33 \mathrm{mg} / \mathrm{dL}$. His urinalysis demonstrated $3+$ blood and protein, with more than 50 red blood cells (RBCs)/hpf and 10-20 WBCs/hpf. Serum creatinine phosphokinase (CPK), uric acid, and phosphorus levels were within normal limits. A bedside bladder scan showed no urine in the bladder. A non-contrast abdominal CT scan revealed normal kidneys with a large amount of ascites (Figure 1).

A Foley catheter was placed and drained three liters of urine. A subsequent liver ultrasound showed complete resolution of the ascites. The patient was diagnosed with a spontaneous bladder rupture causing apparent renal failure and abdominal 


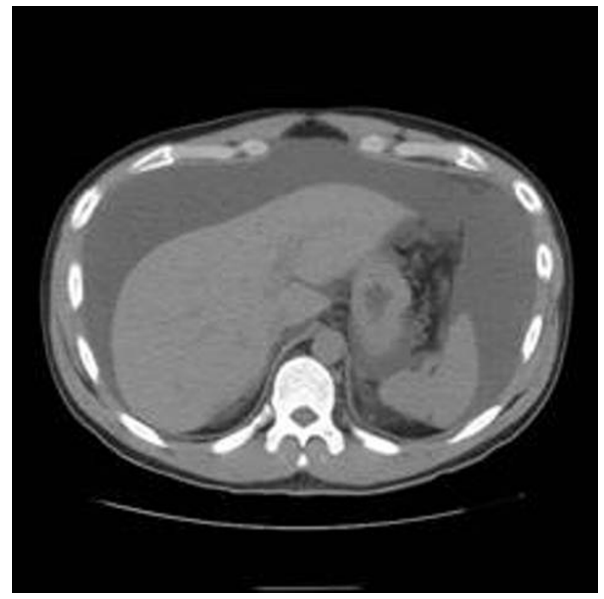

Figure 1. Abdominal CT scan revealing large amount of ascites "urinary ascites". His serum creatinine dropped to $1.2 \mathrm{mg} / \mathrm{dL}$ the next day. He was discharged home with an indwelling Foley catheter. Two weeks after his injury, he had no bladder leak on a voiding cystourethrogram (VCUG; Figure 2).

\section{Discussion}

Rupture of the urinary bladder is an uncommon event. It often is secondary to blunt trauma, pelvic irradiation or bladder wall diseases. ${ }^{1}$ SRUB is very rare and most of the reported cases occurred in association with alcohol intoxication. Late diagnosis and treatment of SRUB is associated with high morbidity and mortality of $50 \%{ }^{2}$

SRUB is caused mainly by bladder overdistention and thinning of the dome wall secondary to a large volume of urine produced by the diuresis effect of alcohol. ${ }^{2}$ In addition, patients with alcohol intoxication have an impaired sensorium and may not feel the urge to void. Moreover, nausea and vomiting associated with alcohol abuse may increase intra-abdominal pressure and the likelihood of bladder rupture in the peritoneal cavity.

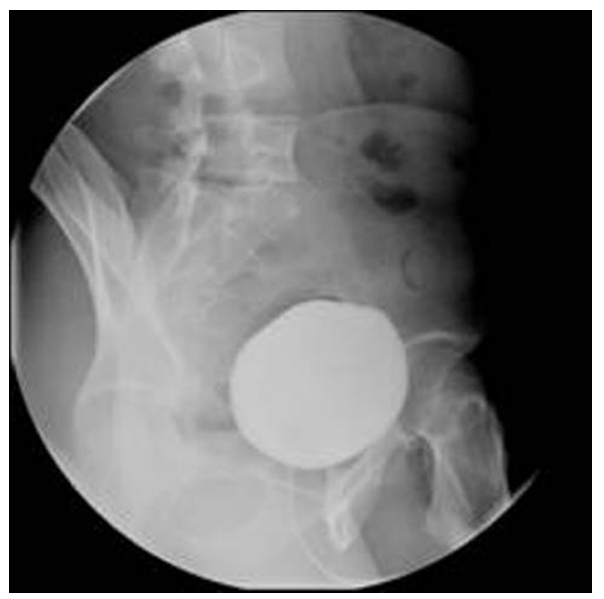

Figure 2. VCUG showing no urine leak from the bladder.

SRUB symptoms usually include severe abdominal pain and macroscopic hematuria ${ }^{1}$, although only microscopic hematuria was present in our patient. The elevation of creatinine in this case likely was caused by direct absorption of urinary creatinine from the ascitic fluid.

SRUB should be suspected in patients with heavy alcohol intoxication, abdominal pain, and low or intermittent urine output. A computed tomography cystogram or retrograde cystography is the procedure of choice to confirm the diagnosis. Paracentesis also can be done and an ascitic fluid creatinine/serum creatinine ratio greater than 1.0 suggests intra-peritoneal urine leak. ${ }^{3}$ Reparative surgery was the standard of care in most of the reported cases. ${ }^{4}$ However, spontaneous healing of the lesion can be achieved if urinary leakage into the peritoneal cavity is prevented. This was accomplished in our patient with bladder catheterization for two weeks. Early diagnosis and treatment of this condition is crucial, since any delay may increase mortality and morbidity secondary to renal failure manifestations. 


\section{References}

${ }^{1}$ Parker H, Hoonpongsimanont W, Vaca F et al. Spontaneous bladder rupture in association with alcoholic binge: a case report and review of the literature. $\mathbf{J}$ Emerg Med. 2009 Nov; 37(4):386-9. PMID: 17976802.

2 Lynn SJ, Mark SD, Searle M. Idiopathic spontaneous bladder rupture in an intoxicated patient. Clinical Nephrol 2003; 60(6):430-432. PMID: 14690262.

${ }^{3}$ Sharma A, Teh B, Morgan DJ, Bell D, Woodhouse $\mathrm{C}$. When ascites is not ascites. Postgrad Med J 2008; 84(995):502-503. PMID: 18940952.
${ }^{4}$ Carmon M, Nissan A, Pappo I, Perlberg S, Seror D, Haskel Y. Spontaneous rupture of the urinary bladder complicated by extensive fascitis: The importance of a high index of suspicion. Urol Int 1994; 52(1):38-40. PMID: 8140678.

Keywords: urinary bladder, spontaneous rupture, alcohol intoxication, case report 